\title{
Aberrant expression of tight junction-related proteins ZO-1, claudin-1 and occludin in synovial sarcoma: an immunohistochemical study with ultrastructural correlation
}

\author{
Steven D. Billings ${ }^{1}$, Shaun V Walsh ${ }^{2}$, Cyril Fisher ${ }^{3}$, Asma Nusrat ${ }^{4}$, Sharon W Weiss ${ }^{4}$, \\ and Andrew L Folpe ${ }^{4}$ \\ ${ }^{1}$ Department of Pathology, Indiana University, Indianapolis, IN, USA; ${ }^{2}$ Department of pathology, Ninewells \\ Hospital, Dundee, UK; ${ }^{3}$ Department of Pathology, The Royal Marsden Hospital, London, UK and \\ ${ }^{4}$ Department of Pathology, Emory University, Atlanta, GA, USA
}

\begin{abstract}
Synovial sarcoma demonstrates epithelial differentiation, either by light microscopy (biphasic synovial sarcoma) or by immunohistochemical/ultrastructural methods only (monophasic) and poorly differentiated synovial sarcoma. Although the glands of synovial sarcoma are known to have tight junction-like structures, far less is known about junction formation in the spindled component of synovial sarcomas. Additionally, it is unknown whether the tight junctions of synovial sarcoma are normally constituted. The tight junction is a multiprotein complex consisting of numerous proteins that include ZO-1, claudin-1 and occludin. A total of 35 cases of synovial sarcoma (13 biphasic, 14 monophasic and eight poorly differentiated) were immunostained for ZO-1, claudin-1 and occludin using commercially available antibodies, heat-induced epitope retrieval and standard avidin-biotin technique. When available, corresponding electron micrographs were reviewed. For five cases, the presence of either an SYT-SSX1 (three cases) or SYT-SSX2 (two cases) gene fusion was known. Positive cases showed particulate membrane staining. The glands of biphasic synovial sarcomas expressed ZO-1 (13/13), claudin-1 (12/13) and occludin (11/13) in a manner identical to normal glandular epithelia, at the apical portion of the lateral membrane. The spindle cells of biphasic synovial sarcomas showed abnormal circumferential membranous expression of ZO-1 (12/13), claudin-1 (6/13) and occludin (3/13). Monophasic synovial sarcomas expressed ZO-1 in a circumferential pattern (13/14) but less often claudin-1 (4/14) or occludin (3/14). Poorly differentiated synovial sarcomas expressed ZO-1 (8/8) and claudin-1 (6/8) but only rarely occludin (2/8). By electron microscopy, recognizable tight junctions were seen only in glands. No correlation was seen between histologic subtype or fusion type and expression of tight junction proteins. We conclude that the glands of biphasic synovial sarcomas show well-organized, true epithelial tight junctions. In contrast, the spindled cells of all synovial sarcomas show significant abnormalities in the expression and localization of tight junction proteins, suggesting partial and/or aberrant epithelial differentiation.
\end{abstract}

Modern Pathology advance online publication, 2 January 2004; doi:10.1038/modpathol.3800042

Keywords: synovial sarcoma; intercellular junctions; zonula occludens; claudin; occludin; tight junctions

Synovial sarcoma is a soft-tissue sarcoma of uncertain histogenesis that is characterized by distinctive morphologic features and a specific translocation between chromosomes $\mathrm{X}$ and 18, resulting in fusion of the $S Y T$ gene to either $S S X 1$ or $S S X 2{ }^{1-4}$ Two distinct subtypes of synovial sarcomas exist, biphasic synovial sarcoma and

Correspondence: AL Folpe, Department of Pathology, H-175, Emory University Hospital, 1364 Clifton Road NE, Atlanta, GA 30322, USA. E-mail: afolpe@emory.edu

Received 20 May 2003; revised 25 August 2003; accepted 17 October 2003; published online 2 January 2004 monophasic synovial sarcoma. ${ }^{5}$ Biphasic synovial sarcomas are composed of both fibroblast-like spindle cells and epithelial cells that often form gland-like structures. Monophasic synovial sarcomas are almost always composed of only the spindle cell component, although extraordinarily rare monophasic epithelial synovial sarcomas do exist. Both biphasic synovial sarcomas and monophasic synovial sarcomas may on occasion show markedly increased cellularity and primitive round cell areas, and such tumors are referred to as 'poorly differentiated synovial sarcomas'. ${ }^{5}$ The histologic features of the spindled component of synovial sarcoma 
overlap significantly with other sarcomas, such as fibrosarcoma and malignant peripheral nerve sheath tumor, and thus the diagnosis of synovial sarcoma requires the demonstration of epithelial differentiation, either by light microscopy, by the immunohistochemical detection of epithelial markers such as cytokeratin or epithelial membrane antigen, or by electron microscopy.

Although earlier ultrastructural studies of synovial sarcoma have documented apparent true epithelial differentiation, including glands with wellformed, normally located tight junctions in biphasic synovial sarcomas and sparse, primitive-appearing intercellular junctions in the spindled cells of monophasic synovial sarcomas and biphasic synovial sarcomas, it is entirely unknown whether these junctions are normally constituted, at the protein level. ${ }^{6-8}$ Recently, however, many of the constitutive proteins of the tight junction have been described. We therefore studied a large series of synovial sarcomas with regard to the expression and localization of a variety of tight junction proteins including the integral structural proteins claudin-1 and occludin and the linker protein ZO-1 by immunohistochemistry. When available, ultrastructural findings were compared with the immunohistochemical findings.

\section{Materials and methods}

Formalin-fixed, paraffin embedded tissue blocks from 35 synovial sarcomas (13 biphasic synovial sarcomas, 14 monophasic synovial sarcomas and 8 poorly differentiated synovial sarcomas) were retrieved from the surgical pathology files of Emory University School of Medicine and the personal consultation files of two of the authors (SWW, CF). All cases were immunostained for claudin-1 (polyclonal, 1:70, Zymed Laboratories, South San Francisco, CA, USA), occludin (Clone OC-3F10, 1:100, Zymed Laboratories, South San Francisco, CA, USA) and ZO-1 (Clone 1, 1:400, Transduction Laboratories, Lexington, KY, USA) using steam heat-induced epitope retrieval and standard avidin-biotin technique. Normal colonic epithelium served as an external positive control for all antibodies, and showed expression of all proteins in a particulate pattern, confined to the apical portion of the lateral epithelial cell membrane. Figure 1 illustrates the normal cellular localization of the various tight junction proteins in a polarized glandular epithelial cell. Normal endothelial cells served as positive internal controls for ZO-1 and occludin; endothelial expression of claudin-1 was more variable. Negative controls consisted of substitution of buffer for the primary antibody. Cases were scored as $3+$ (greater than $50 \%$ positive cells), $2+(25-50 \%$ positive cells), and $1+(5-24 \%$ positive cells). Fisher's exact test was used for statistical evaluation.

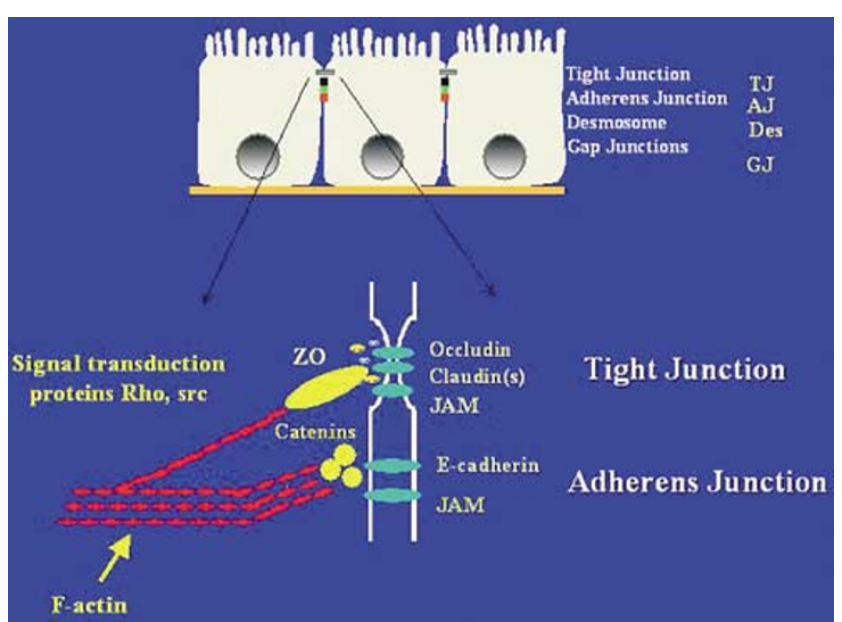

Figure 1 Schematic illustrating three polarized epithelial cells and their intercellular junctions. The tight junction is located most apically on the lateral membrane. The claudins and occludin comprise two of the integral membrane components of the tight junction, where they interact with proteins of the ZO family. This interaction in turn links the tight junction to the actin cytoskeleton.

Corresponding EM prints were available in 14 cases. In five cases, the presence of an SYT-SSX1 (two biphasic synovial sarcomas, one monophasic synovial sarcomas) or SYT-SSX2 (two monophasic synovial sarcomas) had been previously confirmed by molecular genetic methods (data not shown).

All of the hematoxylin-and-eosin-stained sections and the immunohistochemical studies were reviewed by two of the authors (SDB and ALF). A consensus interpretation was reached for all slides. During the course of immunostaining, one case classified as a monophasic synovial sarcoma was found to have a minute focus of subtle, 'occult' gland formation. This case was considered monophasic for purposes of data analysis.

\section{Results}

\section{Biphasic Synovial Sarcomas}

The results for the biphasic synovial sarcomas are summarized in Table 1. The glandular component of biphasic synovial sarcomas expressed ZO-1 in 13 of 13 cases $(100 \%)$, claudin-1 in 12 of 13 cases $(92 \%)$ and occludin-1 in 11 of 13 cases (84\%). In almost all cases, these glands exhibited a staining pattern identical to normal colonic glands, with expression confined to the apical portion of the lateral cell membrane (Figure 2). In one case, however, claudin-1 was expressed only along the basal cell membrane, with apical-lateral expression of ZO-1 and occludin (Figure 3). Overall, normal expression of tight junction proteins was seen in the glands of 10 of 13 biphasic synovial sarcomas, with two cases 
Table 1 Immunohistochemical results

\begin{tabular}{lcrr}
\hline Diagnosis & ZO-1 & Claudin-1 & Occludin \\
\hline Biphasic synovial sarcoma: glands & $13 / 13(1+: 0,2+: 0,3+: 13)$ & $12 / 13(1+: 3,2+: 1,3+: 8)$ & $11 / 13(1+: 1,2+: 4,3+: 6)$ \\
Biphasic synovial sarcoma: spindled cells & $11 / 13(1+: 2,2+: 0,3+: 9)$ & $6 / 13(1+: 2,2+: 3,3+: 1)$ & $3 / 13(1+: 2,2+: 1,3+: 0)$ \\
Monophasic synovial sarcoma & $13 / 14(1+: 0,2+: 3,3+: 11)$ & $4 / 14(1+: 0,2+: 0,3+: 4)$ & $3 / 14(1+: 1,2+: 1,3+: 1)$ \\
Poorly differentiated synovial sarcoma & $8 / 8(1+: 1,2+: 0,3+: 7)$ & $6 / 8(1+: 1,2+: 0,3+: 7)$ & $2 / 8(1+: 1,2+: 0,3+: 7)$ \\
\hline
\end{tabular}
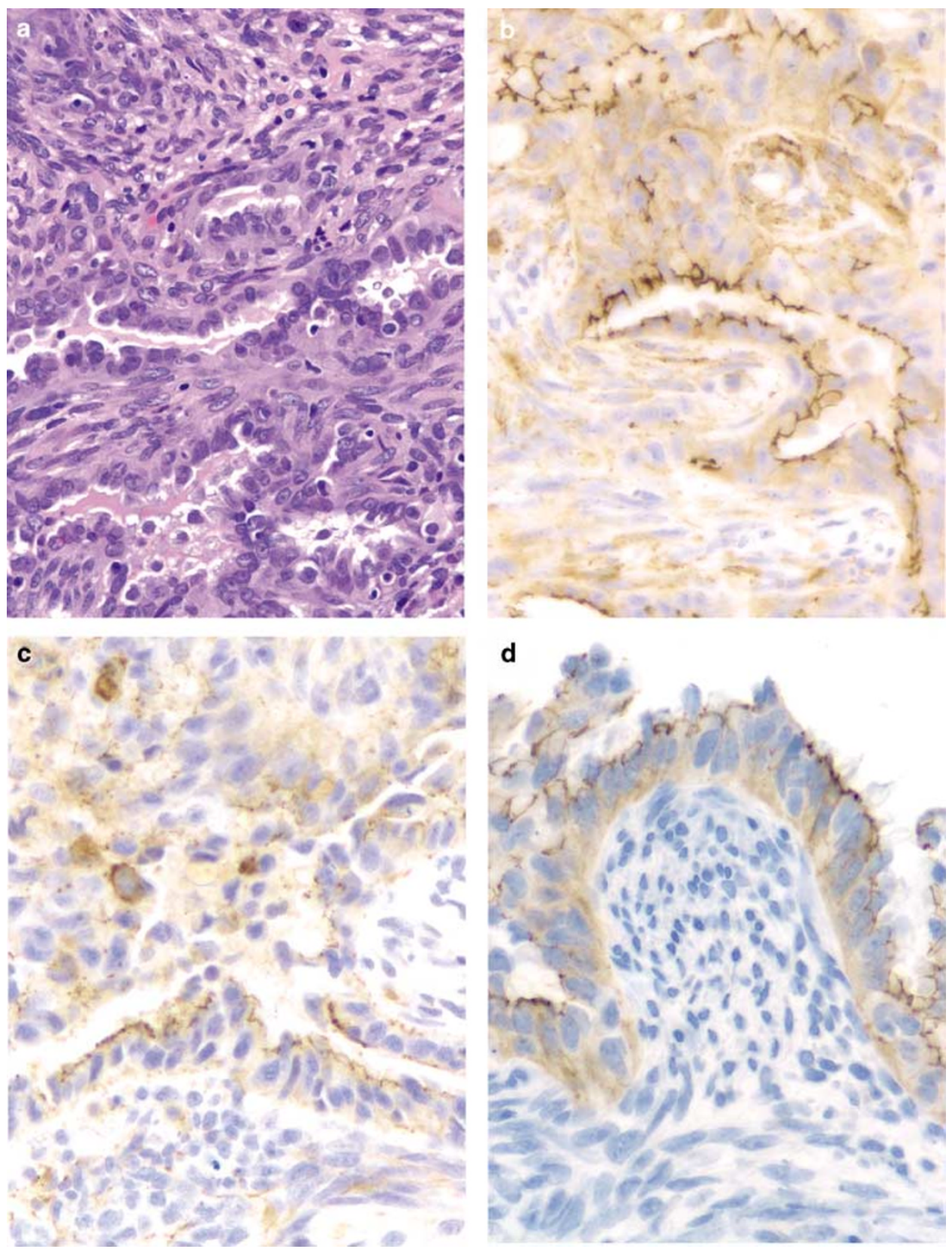

Figure 2 Biphasic synovial sarcoma, showing both glandular and spindle cell components (a). The glands of biphasic synovial sarcomas usually show a normal pattern of tight junction protein expression, with expression of ZO-1 (a), claudin-1 (b), and occludin (c) at the apical-lateral portion of the cell membrane. In contrast, abnormal, nonpolarized, circumferential expression of ZO-1 and claudin-1 is commonly seen in the spindled cells. Occludin expression, however, is almost always restricted to cells with clear glandular differentiation. 

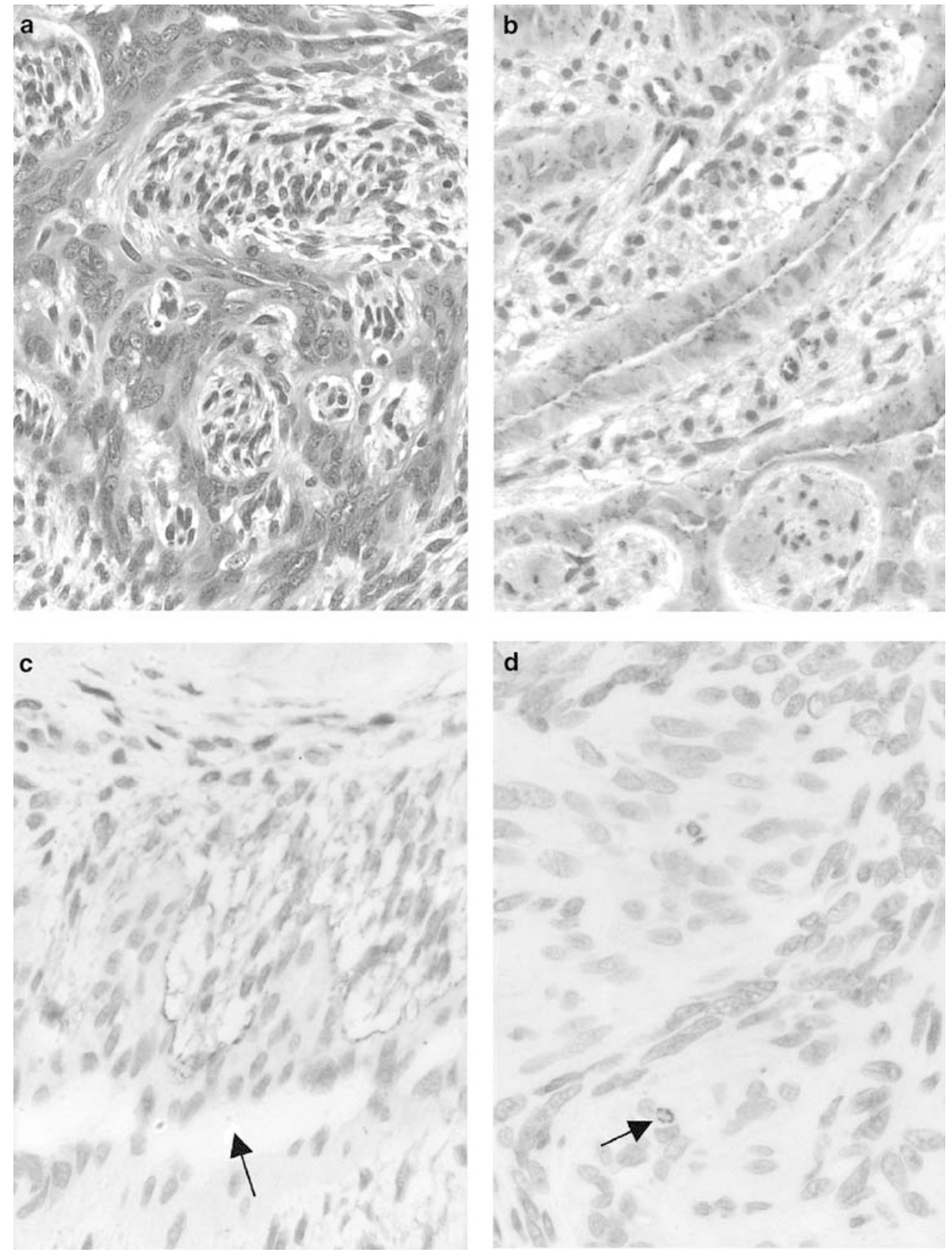

Figure 3 Biphasic synovial sarcoma, showing an unusual serpiginous pattern of glandular differentiation (a). As would be expected in normal glands, ZO-1 was expressed along the apical-lateral membrane of the glandular cells (b). In contrast, however, claudin-1 was expressed exclusively along the basal cell membrane, adjacent to the spindled cells and away from the apparent gland lumen (arrow) (c). The highly abnormal tight junction structure of these glands is further suggested by the absence of occludin expression (c). Positive capillary endothelium is present, serving as an internal positive control (arrow).

lacking only occludin and one case lacking only claudin-1.

The spindle cell component of biphasic synovial sarcomas showed circumferential (nonpolarized) membranous expression of ZO-1, claudin-1 and occludin in 12 of $13(85 \%)$, six of $13(46 \%)$ and three of $13(16 \%)$ of cases, respectively. In contrast to the glandular elements, the spindled cells expressed all three tight junction proteins in only two of 13 cases, with five cases expressing only
ZO-1 and claudin-1, three cases expressing only $\mathrm{ZO}-1$, and three cases lacking expression of any tight junction proteins in the spindled cells.

\section{Monophasic Synovial Sarcoma}

The results for the monophasic synovial sarcomas are summarized in Table 1 . The spindled cell of monophasic synovial sarcomas expressed ZO-1, 
claudin-1 and occludin in 13 of 14 cases (93\%), four of 14 cases $(27 \%)$ and three of 14 cases $(21 \%)$, respectively (Figure 4). Monophasic synovial sarcomas expressed all three tight junction proteins in two cases, ZO-1 and claudin-1 in two cases, ZO-1 only in nine cases, and no tight junction proteins in one case. As in the spindled component of biphasic synovial sarcomas, all tight junction proteins were expressed in a circumferential membranous pattern. In one case previously classified as monophasic synovial sarcomas, occludin immunostaining revealed 'occult' gland formation, suggesting that this case was in fact a biphasic synovial sarcoma.

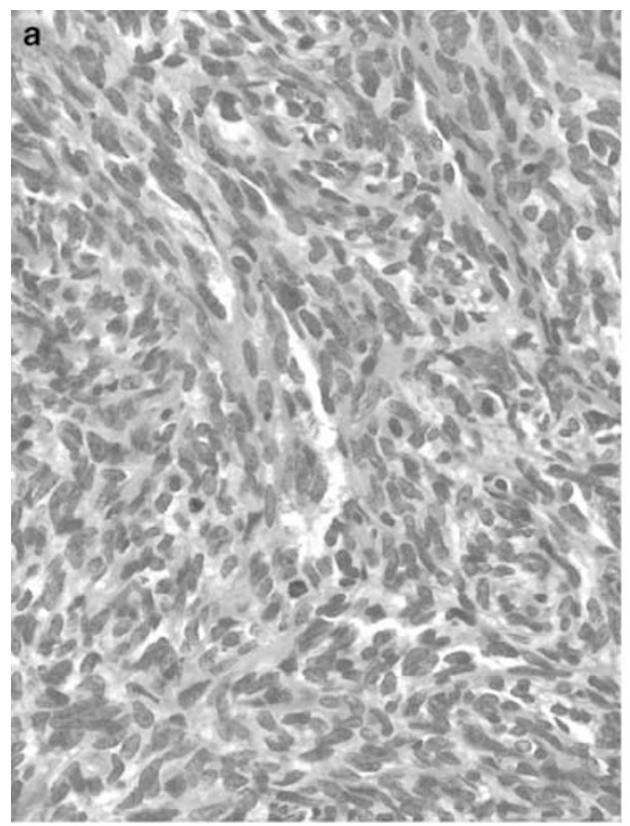

\section{Poorly Differentiated Synovial Sarcoma}

The results for the poorly differentiated synovial sarcomas are summarized in Table 1. Expression of ZO-1, claudin-1 and occludin was seen in eight of eight $(100 \%)$, six of eight $(75 \%)$ and two of eight $(25 \%)$ of cases, respectively. Poorly differentiated synovial sarcomas expressed all three tight junction proteins in two cases, ZO-1 and claudin-1 in four cases, and ZO-1 only in two cases. In all positive cases, only nonpolarized membranous staining was seen (Figure 5). 'Occult' gland formation was not observed in any case.

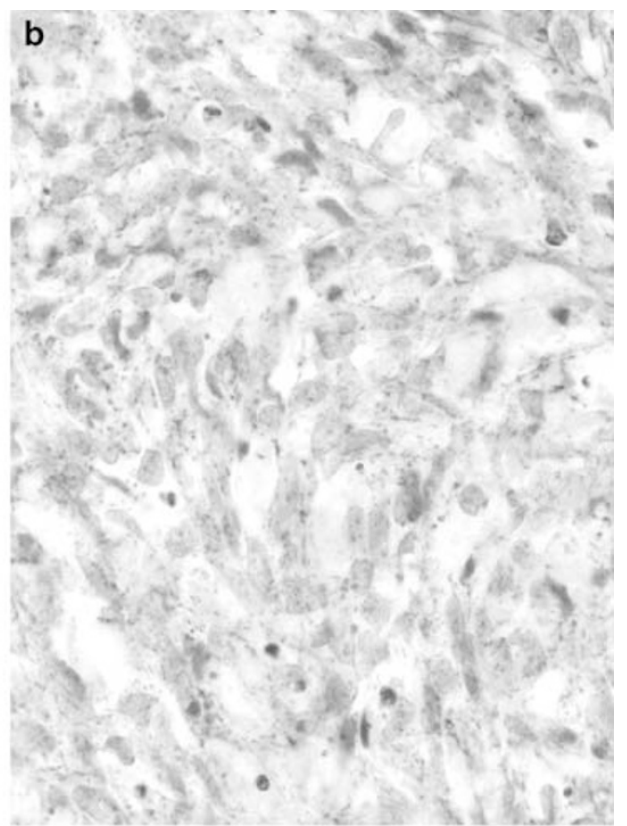

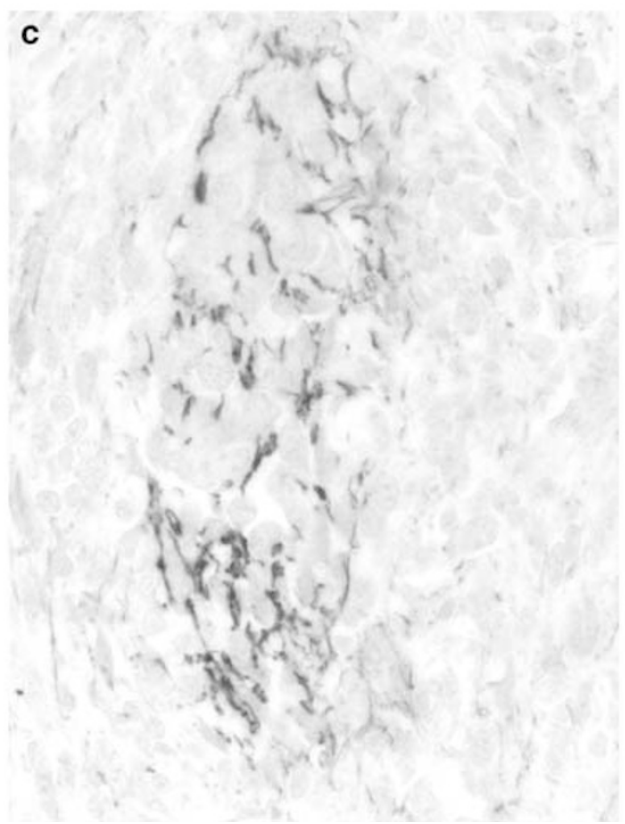

Figure 4 Monophasic synovial sarcoma (a) showing nonpolarized expression of ZO-1 (b) and claudin-1 (c). In this particular case, claudin-1 expression was seen primarily in clusters of vaguely epithelioid cells, suggesting the possibility of focal early glandular differentiation. Occludin was not, however expressed by these cells (data not shown). 

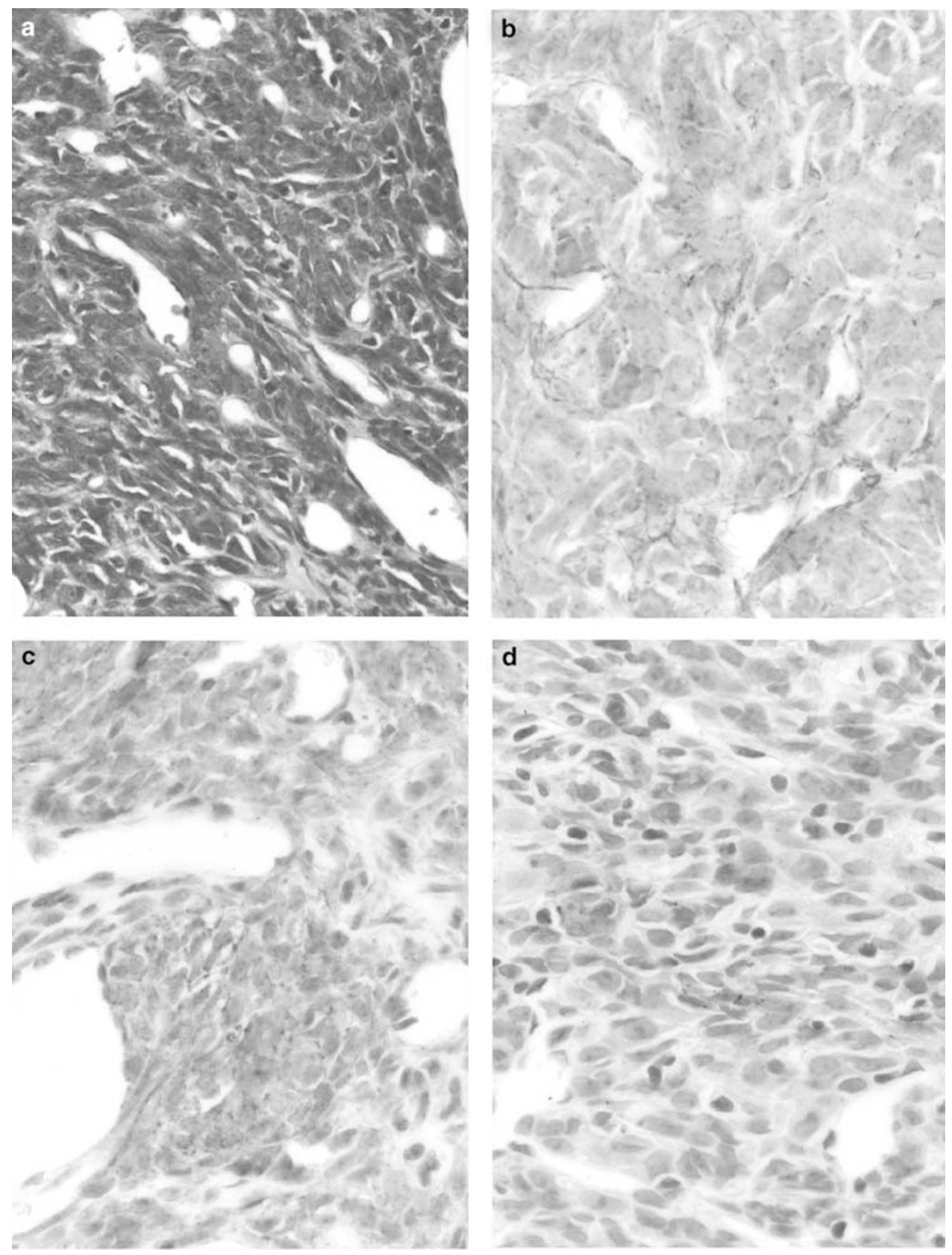

Figure 5 Poorly differentiated synovial sarcoma, characterized by marked hypercellularity and a 'malignant hemangiopericytoma'-like appearance (a). As in the spindled cell of biphasic synovial sarcomas and monophasic synovial sarcomas, expression of ZO-1 (b) and claudin-1 (c) was seen in a nonpolarized fashion, suggestive of aberrant epithelial differentiation. Interestingly, however, occludin expression was seen in a subset of poorly differentiated synovial sarcomas, suggesting a paradoxically higher level of epithelial differentiation (d).

\section{Expression of Tight Junction Proteins by Fusion Type}

The two biphasic synovial sarcomas with known SYT-SSX1 showed expression of all three tight junction proteins in their glands. In contrast, although ZO-1 was focally positive in the spindled cells of one case, all other markers were negative. The monophasic synovial sarcomas with known $S Y T-S S X 1$ and the two monophasic synovial sarcomas with known SYT-SSX2 all expressed only ZO-1.

\section{Electron Microscopy}

In all cases of biphasic synovial sarcomas with available electron microscopy prints, morphologically normal tight junctions were visible at the apical portion of the lateral cell membrane in glandular cells (Figure 6a). Recognizable tight junctions were not identified in the spindled cells in biphasic synovial sarcomas, monophasic synovial sarcomas or poorly differentiated synovial sarcomas. 

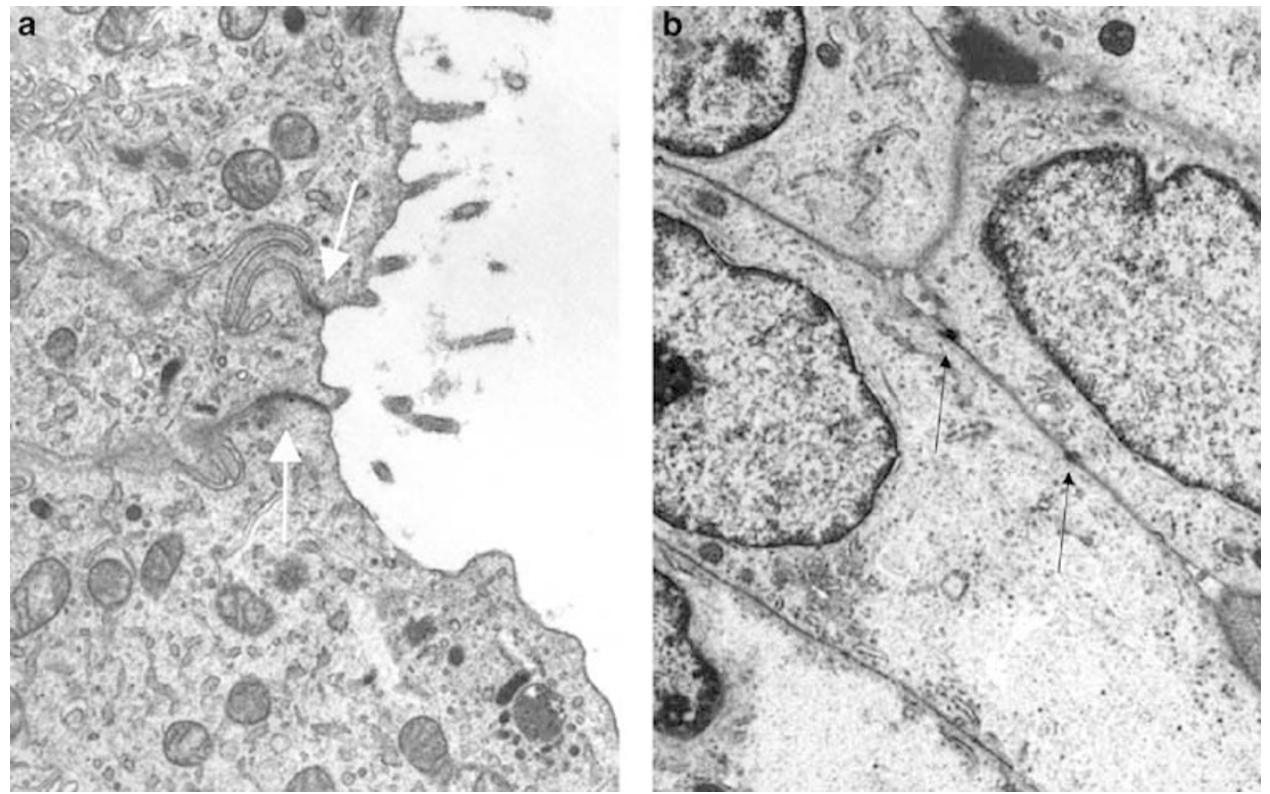

Figure 6 Electron micrographs of biphasic (a) and monophasic (b) synovial sarcoma cells. Normally located, normal appearing tight junctions can be clearly seen at the apical portion of the lateral membrane at the junction of these three polarized glandular cells in this biphasic synovial sarcomas. In contrast, the spindled cells of this monophasic synovial sarcomas show only two randomly distributed, primitive intercellular junctions, of uncertain type.

Instead, only rare membrane densities were identified, with an apparently random distribution along the plasmalemmal membrane (Figure 6b).

\section{Discussion}

In epithelial and endothelial cells, tight junctions limit the exchange of lipids between the apical and basal membranes, thereby defining membrane polarity, forming an intercellular seal, and regulating the paracellular transport of water, solutes and cells. ${ }^{9}$ Although tight junctions in epithelial tissue were once thought to provide an impermeable 'tight' barrier, it is now recognized that they play a dynamic role in paracellular solute transport, cell polarity and cell-cell adhesion rather than functioning merely as a static barrier. Over the last few years, the molecular constituents of tight junctions have been isolated. These include integral transmembrane proteins such as the claudins, occludin and junctional adhesion molecule and scaffolding proteins such as ZO-1, ZO-2 and ZO-3. ${ }^{10}$ Through interactions of the scaffolding proteins with the actin cytoskeleton, tight junctions also appear to play a critical role in signal transduction. ${ }^{9}$

Occludin was the first tight junction-specific integral membrane protein to be identified and is thought to be both a structural and functional component of the tight junction. ${ }^{11,12}$ While occludin has been identified in most epithelial tight junctions, at both the gene and protein level, its role in assembly and maintenance of tight junctions is still undefined. ${ }^{13}$ Although essentially all normal tight junctions appear to contain occludin, there are experimental data in knockout mouse and stem cell models that suggest occludin may not always be required for the formation of tight junctions. ${ }^{13}$ The claudins are a family of approximately 18 homologous proteins that play a key role in determining tight junction structure and permeability in both endothelial and epithelial cells. ${ }^{10}$ Interestingly, the members of the claudin family appear to be differentially expressed in tissues. Claudin-1 expression is relatively widespread among epithelia, whereas claudin-3 is primarily expressed in lung and liver epithelia and claudin-5 in endothelial cells. ${ }^{10}$ ZO-1 (zonula occludens-1) serves as a scaffolding protein that links the transmembrane tight junction proteins to cytoplasmic proteins and the actin cytoskeleton. ${ }^{14,15}$ However, ZO-1 expression is not confined to the tight junction ${ }^{16}$ and it has recently been shown to be involved in the formation of the adherens junction, as well. ${ }^{17}$ Accordingly, ZO-1 is expressed in a variety of nonepithelial cell types including Schwann cells, dermal fibroblasts, astrocytes, and the C6 glioma, S-180 (sarcoma) and P3 myeloma cell lines. ${ }^{18-20}$

Despite its name, it is now well established that synovial sarcoma has no relationship to normal synovium, a tissue derived from nonepithelial, histiocyte-like synoviocytes. ${ }^{7,8,21}$ As is well known, synovial sarcoma is characterized by partial epithelial differentiation, recognizable with routine microscopy in biphasic synovial sarcomas, and with the assistance of immunohistochemical and 
8

ultrastructural techniques in monophasic synovial sarcomas and poorly differentiated synovial sarcomas. Ultrastructurally, the glands of biphasic synovial sarcomas appear to be composed of relatively well-differentiated epithelial cells, in that they show true lumen formation, morphologically and spatially normal junctional complexes including tight junctions, adherens junctions and desmosomes, and basal lamina. ${ }^{6,7}$ Despite their intimate admixture with the glandular cells, the spindled cells of biphasic synovial sarcomas (and monophasic synovial sarcomas) show little ultrastructural evidence of epithelial differentiation, with only primitive cell junctions of uncertain type. ${ }^{6,7}$

Our data confirm and extend prior ultrastructural studies with regard to the presence and location of true tight junctions in synovial sarcoma. In agreement with ultrastructural findings, the glandular elements of biphasic synovial sarcomas appear to form normally constituted tight junctions, as evidenced by normal expression of occludin, claudin-1 and ZO-1 at the apical portion of the lateral cell membrane, in over $75 \%$ of cases. Interestingly, however, two of 13 (15\%) of biphasic synovial sarcomas lacked occludin expression, suggesting a significant aberration in tight junction formation. As noted above, there is some suggestion that occludin is not necessary for tight junction formation, although all known glandular epithelia express occludin. The significance of one biphasic synovial sarcoma that expressed occludin and ZO-1, but not claudin, is less clear. Although this may indicate an abnormality in tight junction formation, it might also reflect expression of another member of the claudin family. ${ }^{10}$ Study of additional cases of biphasic synovial sarcoma with antibodies to other claudins will be necessary to test this hypothesis. The significance of the basal pattern of claudin-1 expression seen in one case is unclear, but could represent a derangement in cellular polarization and defective tight junction function. Expression of other intercellular adhesion molecules, such as E-cadherin and $\mathrm{N}$-cadherin, has been documented recently in almost all biphasic synovial sarcomas and in significant subsets of monophasic and poorly differentiated synovial sarcomas..$^{2,23}$

In contrast to the relatively normal expression of tight junction proteins in the glands of biphasic synovial sarcomas, the spindled cells of biphasic synovial sarcomas, monophasic synovial sarcomas and poorly differentiated synovial sarcomas show profound abnormalities in tight junction formation, in most cases. In evaluating expression of tight junction proteins, it is important to remember that ZO-1 has functions outside of the tight junction, specifically in the adherens junction. Conceivably, therefore, in those cases in which the spindled cells express only ZO-1, the expression of ZO-1 may not reflect tight junction formation at all. Expression of ZO-1 and claudin-1, with or without occludin, was seen in the spindled cells of $54 \%$ of biphasic synovial sarcomas, $29 \%$ of monophasic synovial sarcomas, and $75 \%$ of poorly differentiated synovial sarcomas and it seems reasonable to assume that the cells in these cases form tight junction. It is impossible to know to what extent these are functionally normal tight junction, although the absence of occludin in most cases strongly suggests abnormalities. The localization of these junctions is, however, clearly aberrant. Circumferential (nonpolarized) expression of tight junction proteins, as seen in almost every case, is not observed in any normal cell, to the best of our knowledge. Most likely this pattern of expression represents partial and/or abnormal epithelial differentiation in all cells in synovial sarcomas. Although we had initially hypothesized that the spindled cells of biphasic synovial sarcomas would be 'better differentiated' than those of monophasic synovial sarcomas, with respect to tight junction protein expression, our data show this not to be the case. We did not observe a statistically significant difference in expression of any of the individual tight junction proteins when comparing the spindled cells of biphasic synovial sarcomas, monophasic synovial sarcomas and poorly differentiated synovial sarcomas. Similarly, no significant differences were seen in the percentages of biphasic synovial sarcomas, monophasic synovial sarcomas or poorly differentiated synovial sarcomas showing simultaneous expression of the three tight junction proteins studied.

Synovial sarcomas contain a characteristic and specific $\mathrm{t}(\mathrm{X} ; 18)$ that results in the fusion of the $S Y T$ gene from chromosome 18 with either the $S S X 1$ or SSX2 genes from the X chromosome ${ }^{1-4}$ The SYTSSX1 subtype is present in approximately twothirds of synovial sarcomas with the remainder having the SYT-SSX2 subtype. ${ }^{24-26}$ The translocation subtype corresponds to morphologic subtype. Biphasic synovial sarcomas exclusively have the $S Y T-S S X 1$ subtype, whereas in monophasic synovial sarcomas both subtypes are present in roughly equal numbers. This has led to the suggestion that the SYT-SSX1 fusion may play a role in glandular morphogenesis in synovial sarcomas. ${ }^{26}$ Although we had hypothesized that the spindled cells of cases with SYT-SSX1 fusions might show higher levels of tight junction protein expression, our admittedly limited data do not support this. Specifically, we found expression of ZO-1 in two of three SYT-SSX1 cases and both SYT-SSX2 cases, with no expression of occludin or claudin-1 in any of these five cases. Obviously, however, only limited conclusions can be drawn from our relatively small number of cases, and it is conceivable that study of larger number of cases might reveal a significant association between fusion type and tight junction formation.

Is there a role for tight junction proteins in the diagnosis of synovial sarcoma? Although we had initially hoped that this might be the case, it is clear that the sensitivity of these markers is considerably 
less than that of conventional markers, such as cytokeratins and epithelial membrane antigen. Additionally, we have observed expression of ZO-1 and claudin-1 in a subset of those sarcomas that enter the differential diagnosis of synovial sarcoma, such as malignant peripheral nerve sheath tumors and malignant solitary fibrous tumors (data not shown). We have also previously reported claudin-1 expression in perineurial cells and perineuriomas. ${ }^{27}$

In summary, we examined for the first time the composition and localization of tight junctions in synovial sarcomas. Although the glandular elements of biphasic synovial sarcomas show relatively normal tight junctions, as compared with normal glandular epithelia, the spindled cells of all types of synovial sarcoma display profound abnormalities in the expression and localization of ZO-1, claudin-1 and occludin. Additional study of the structure and function of tight junctions in synovial sarcoma may provide further insight into the biology and behavior of this unique sarcoma with epithelial differentiation.

\section{References}

1 Argani P, Zakowski MF, Klimstra DS, et al . Detection of the SYT-SSX chimeric RNA of synovial sarcoma in paraffin-embedded tissue and its application in problematic cases. Mod Pathol 1998;11:65-71.

2 Crew AJ, Clark J, Fisher C, et al. Fusion of SYT to two genes, SSX1 and SSX2, encoding proteins with homology to the Kruppel-associated box in human synovial sarcoma. EMBO J 1995;14:2333-2340.

3 de Leeuw B, Suijkerbuijk RF, Olde Weghuis D, et al. Distinct Xp11.2 breakpoint regions in synovial sarcoma revealed by metaphase and interphase FISH: relationship to histologic subtypes. Cancer Genet Cytogenet 1994;73:89-94.

4 Ladanyi M, Woodruff JM, Scheithauer BW, et al. Re: O’Sullivan MJ, Kyriakos M, Zhu X, Wick MR, Swanson PE, Dehner LP, Humphrey PA, Pfeifer JD: malignant peripheral nerve sheath tumors with $t(X ; 18)$. A pathologic and molecular genetic study. Mod Pathol 2001;14:733-737.

5 Weiss SW, Goldblum JR. Enzinger and Weiss's Soft Tissue Tumors. 4th edn. St. Louis: Mosby: St Louis, MO, 2001.

6 Ordonez NG, Mahfouz SM, Mackay B. Synovial sarcoma: an immunohistochemical and ultrastructural study. Hum Pathol 1990;21:733-749.

7 Fisher C. Synovial sarcoma: ultrastructural and immunohistochemical features of epithelial differentiation in monophasic and biphasic tumors. Hum Pathol 1986;17:996-1008.

8 Smith ME, Fisher C, Wilkinson LS, et al. Synovial sarcoma lack synovial differentiation. Histopathology 1995;26:279-281.

9 Nusrat A, Parkos CA, Verkade P, et al. Tight junctions are membrane microdomains. J Cell Sci 2000;113: 1771-1781.

10 Heiskala M, Peterson PA, Yang Y. The roles of claudin superfamily proteins in paracellular transport. Traffic 2001;2:93-98.
11 Furuse M, Hirase T, Itoh M, et al. Occludin: a novel integral membrane protein localizing at tight junctions. J Cell Biol 1993;123:1777-1788.

12 Ando-Akatsuka Y, Saitou M, Hirase T, et al. Interspecies diversity of the occludin sequence: cDNA cloning of human, mouse, dog, and rat-kangaroo homologues. J Cell Biol 1996;133:43-47.

13 Saitou M, Furuse M, Sasaki H, et al. Complex phenotype of mice lacking occludin, a component of tight junction strands. Mol Biol Cell 2000;11:41314142.

14 Furuse M, Itoh M, Hirase T, et al. Direct association of occludin with ZO-1 and its possible involvement in the localization of occludin at tight junctions. J Cell Biol 1994;127:1617-1626.

15 Fanning AS, Jameson BJ, Jesaitis LA, et al. The tight junction protein ZO-1 establishes a link between the transmembrane protein occludin and the actin cytoskeleton. J Biol Chem 1998;273:29745-29753.

16 Saitou M, Ando-Akatsuka Y, Itoh M, et al. Mammalian occludin in epithelial cells: its expression and subcellular distribution. Eur J Cell Biol 1997;73: 222-231.

17 Itoh M, Nagafuchi A, Yonemura S, et al. The 220-kD protein colocalizing with cadherins in non-epithelial cells is identical to ZO-1, a tight junction-associated protein in epithelial cells: cDNA cloning and immunoelectron microscopy. J Cell Biol 1993;121:491-502.

18 Howarth AG, Hughes MR, Stevenson BR. Detection of the tight junction-associated protein ZO-1 in astrocytes and other nonepithelial cell types. Am J Physiol 1992;262:C461-C469.

19 Howarth AG, Singer KL, Stevenson BR. Analysis of the distribution and phosphorylation state of ZO-1 in MDCK and nonepithelial cells. J Membr Biol 1994;137:261-270.

20 Howarth AG, Stevenson BR. Molecular environment of ZO-1 in epithelial and non-epithelial cells. Cell Motil Cytoskeleton 1995;31:323-332.

21 Miettinen M, Virtanen I. Synovial sarcoma-a misnomer. Am J Pathol 1984;117:18-25.

22 Pelmus M, Guillou L, Hostein I, et al. Monophasic fibrous and poorly differentiated synovial sarcoma: immunohistochemical reassessment of $60 \mathrm{t}(\mathrm{X} ; 18)(\mathrm{SYT}$ SSX)-positive cases. Am J of Surg Pathol 2002;26: 1434-1440.

23 Laskin WB, Miettinen M. Epithelial-type and neuraltype cadherin expression in malignant noncarcinomatous neoplasms with epithelioid features that involve the soft tissues. Arch Path Lab Med 2002;126: 425-431.

24 Ladanyi M. Fusions of the SYT and SSX genes in synovial sarcoma. Oncogene 2001;20:5755-5762.

25 Ladanyi M, Antonescu CR, Leung DH, et al. Impact of SYT-SSX fusion type on the clinical behavior of synovial sarcoma: a multi-institutional retrospective study of 243 patients. Cancer Res 2002;62: 135-140.

26 Antonescu CR, Kawai A, Leung DH, et al. Strong association of SYT-SSX fusion type and morphologic epithelial differentiation in synovial sarcoma. Diagn Mol Pathol 2000;9:1-8.

27 Folpe AL, Billings SD, McKenney JK, et al. Expression of claudin-1, a recently described tight junctionassociated protein, distinguishes soft tissue perineurioma from potential mimics. Am J Surg Pathol 2002;26:1620-1626. 